\title{
Une momie à la Bnu
}

\section{Julien Collonges et Daniel Bornemann}

\section{(2) OpenEdition}

\section{Journals}

Édition électronique

URL : https://journals.openedition.org/rbnu/3359

DOI : $10.4000 /$ rbnu.3359

ISSN : 2679-6104

\section{Éditeur}

Bibliothèque nationale et universitaire de Strasbourg

\section{Édition imprimée}

Date de publication : 1 novembre 2010

Pagination : 5

ISSN : 2109-2761

\section{Référence électronique}

Julien Collonges et Daniel Bornemann, « Une momie à la Bnu », La Revue de la BNU [En ligne], 2 | 2010, mis en ligne le 01 novembre 2010, consulté le 09 août 2021. URL : http://journals.openedition.org/ rbnu/3359; DOI : https://doi.org/10.4000/rbnu.3359

\section{(c) (i) (2) (-)}

La Revue de la BNU est mise à disposition selon les termes de la Licence Creative Commons Attribution - Pas d'Utilisation Commerciale - Partage dans les Mêmes Conditions 4.0 International. 


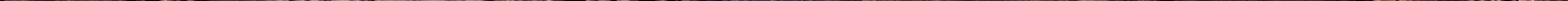




\section{UNE MOMIE À LA BNU}

Entretien avec Daniel Bornemann

Rares sont ceux qui disent l'avoir déjà vue, mais beaucoup à la BNU croient le savoir : il y aurait une momie dans les murs de la bibliothèque. Daniel Bornemann, responsable des fonds égyptologiques et des collections de papyrus de la BNU, nous en dit un peu plus.

\section{Daniel Bornemann, il y a donc une momie égyptienne dans les collections de la BNU? \\ C'est exact.}

\section{Où se trouve-t-elle ?}

Dans la chambre forte qui contient les trésors égyptologiques de la $\mathrm{BNU}$, et notamment sa très importante collection de papyrus.

\section{Dans quel état est-elle?}

Apparemment en mauvais état, comme la plupart des momies, qui sont le plus souvent assez mal conservées. Mais il serait sans doute intéressant d'examiner de plus près son état réel. Or la momie est conservée et présentée dans une grande boîte vitrée en bois, fermée par une serrure dont nous n'avons pas la clef. Personne n'a donc pu l'examiner depuis plusieurs dizaines d'années. Ce que l'on peut dire c'est que la momie a été manifestement placée dans une caisse trop petite pour sa taille : elle est très à l'étroit et un peu sens dessus dessous. Elle est de toute évidence en plusieurs morceaux. Le crâne, en tous cas, est détaché du corps. On reconnaît un bras et une main et ce qui semble être le pelvis, et l'on aperçoit dans la cage thoracique les épices et plantes qui ont servi à l'embaumement.

\section{On ne peut donc pas déterminer sa taille exacte ?}

Non. La caisse où elle repose mesure environ 1,30 m. Mais pour connaître la taille de la momie, il faudrait la reconstituer. On peut seulement affirmer que c'est la taille d'un être humain adulte.

\section{Quelle est sa provenance?}

L'Égypte, c'est certain. Mais pour le reste, on n'en sait malheureusement rien.

\section{Par quel biais s'est-elle retrouvée dans les collections de la bibliothèque ?}

On n'a acquis un peu plus de certitudes que très récemment. Mon collègue Christophe Didier, au cours de son travail sur Julius Euting, qui fut administrateur de la BNU au début du $\mathrm{XX}^{e}$ siècle, a découvert dans un article de l'époque que ce dernier, dans son testament, avait légué un certain nombre d'objets à la BNU, ainsi qu'à d'autres institutions 


\section{Le testament d'Euting précise qu'une "caisse à ossements" est léguée à l'Institut des antiquités de l'université}

strasbourgeoises. Une liste très succincte précisait qu'il s'agissait d'objets égyptologiques et mentionnait une momie. En consultant nos dossiers d'archives sur les dons et legs faits à la BNU, nous avons alors retrouvé une photo du testament original d'Euting qui précise le contenu du legs : une collection de poids monétaires en verre et une "Knochenkiste ", une " caisse à ossements " qui, elle, n'est pas léguée à la BNU - ou à son ancêtre la Kaiserliche Universitäts- und Landesbibliothek - mais à l'Institut des antiquités de l'université. La piste semblait donc s'arrêter là. En même temps, l'appellation un peu brutale de "Knochenkiste " correspondait fort bien à la momie conservée à la BNU. Et en fouillant plus avant dans le dossier sur le legs d'Euting, nous avons trouvé un autre document qui précise que si la caisse à ossements est léguée à l'Institut des antiquités actuel Institut d'égyptologie, certains objets, dont les " grandes antiquités ", peuvent rester en dépôt à la bibliothèque del'université, donc, à l'époque, à la BNU. Une lettre d'un des administrateurs ayant succédé à Euting confirme que ces " grandes antiquités ", qui comprenaient, outre la momie, un petit autel votif, furent bien léguées à l'université. Cela expliquerait que ces objets, déposés à la BNU mais appartenant à l'université, n'aient été ni inventoriés ni cotés.

\section{Qui était ce Julius Euting?}

Il fut d'abord bibliothécaire à la Kaiserliche Universitäts- und Landesbibliothek avant d'en devenir le directeur en 1900 (voir à ce sujet l'article de C. Didier p. 105). C'était aussi un orientaliste, avant tout intéressé par le monde arabe. Mais le monde arabe comprenant aussi l'Égypte, il s'intéressa également aux antiquités égyptiennes, aux papyrus, aux ostraca, etc. Il était par ailleurs lié au Deutsches Papyruskartell, une des principales sociétés d'égyptologie actives à Strasbourg à cette époque, qui avait son siège à la BNU.

\section{Sait-on pour quelle raison Euting a ramené cette momie à Strasbourg ? Était-elle destinée à devenir un objet d'étude ?}

Rien ne le précise dans les documents que nous avons retrouvés. Mais mon hypothèse personnelle est que cette momie n'a pas tant été rapportée pour elle-même que pour en exploiter les cartonnages. Ces cartonnages, constitués de plusieurs couches de papyrus de récupération, souvent porteurs d'inscriptions, étaient collés les uns sur les autres pour être en quelque sorte recyclés dans l'embaumement des momies. Ainsi assemblés, ils permettaient de recouvrir la momie d'une coque que l'on pouvait peindre, dorer ou orner de plaques de cuivre. Il est donc possible que la momie ait avant tout été rapportée pour étudier les textes contenus dans ces papyrus de récupération.

\section{Existe-t-il des indices de cette dernière hypothèse ? Des traces de cet intérêt pour les cartonnages?}

Il y en a plusieurs, oui. La BNU possède une caisse de débris de papyrus issus de cartonnages de momies, rarement porteurs d'écritures, et qui n'ont donc pas été exploités au sein de la bibliothèque des papyrus. En outre, la momie étant partiellement désarticulée et démembrée, il est possible que cela soit dû aux manipulations destinées à retirer les cartonnages. Il existe aussi dans nos collections une bandelette de momie, relativement longue et porteuse d'écritures, et qui pourrait être en rapport avec notre momie, même si rien ne nous permet de l'affirmer à ce jour. Enfin, les fonds de papyrus de la BNU comportent des fragments, issus de la collection de la Wissenschaftliche Gesellschaft - une autre société d'égyptologie strasbourgeoise de l'époque dont la forme indique clairement qu'ils sont issus de cartonnages de momies. 


\section{Que sait-on du personnage momifié ?}

Pas grand-chose, si ce n'est qu'il s'agirait d'une femme, comme semble l'attester le masque mortuaire accompagnant la momie qui ne comporte pas la barbe caractéristique des momies masculines. Mais des égyptologues pourraient probablement nous en dire beaucoup plus, notamment en interprétant le masque ou d'autres indices. D'autant plus que l'Alsace est une région riche de savoirs sur les momies. Geneviève Fuchs, responsable des collections égyptologiques du Musée d'histoire naturelle et d'ethnographie de Colmar, a par exemple effectué des recherches poussées sur les momies conservées au musée. En outre, il existe d'autres momies conservées à Strasbourg : l'Institut d'égyptologie de l'Université de Strasbourg en possède une et l'Institut d'anatomie de l'hôpital universitaire de Strasbourg abrite toute une collection de têtes de momies. Le champ des investigations possibles est donc vaste.

\section{La BNU conserve-t-elle d'autres objets ou documents liés aux momies?}

Oui, beaucoup. Il y a par exemple ce papyrus expliquant les différentes méthodes de momification qui a été tout récemment exposé à Stuttgart. Il y aussi un masque de momie, de style gréco-latin et un petit morceau de momie dont l'étiquette mentionne « Morceau de la momie de Tübingen ". La BNU possède également plus de 250 étiquettes de momies, ces morceaux de bois comportant diverses informations relatives aux corps embaumés (nom et origine du défunt, méthode de momification, destination), et des " livres des morts ", ces livres qui accompagnaient les momies dans leur dernier voyage.

\section{Les chercheurs, notamment les égyptologues et papyrologues qui fréquentent la BNU, ne se sont-ils jamais intéressés à cette momie ?}

À ma connaissance, non. En tous cas, je n'ai pas trouvé trace, dans les archives de la BNU de la seconde moitié $\mathrm{du} \mathrm{XX}^{\mathrm{e}}$ siècle, d'une visite d'un chercheur consacrée à la momie. Celle-ci est sans doute exploitable par les égyptologues, mais il est probable qu'elle faisait à l'origine partie d'un ensemble de documents et d'objets présentant des difficultés particulières que les chercheurs laissèrent dans un premier temps de côté pour se consacrer d'abord aux éléments plus aisément interprétables. Aujourd'hui encore, les égyptologues compétents se partagent beaucoup entre l'enseignement et des recherches à partir des livres, d'une part, et d'autre part des fouilles sur le terrain, en Egypte, qui leur permettent d'exhumer des objets nombreux et sans doute plus intéressants, pour l'instant, que la momie de la BNU. L'étude de ces objets provenant d'Égypte prime encore sur les quelques autres conservés ça et là et dont la difficulté d'interprétation a retardé l'examen.

\section{Une dernière chose : les momies sont souvent associées, dans l'imaginaire et la littérature, à des histoires fantastiques, et l'on évoque à leur propos des récits étranges de malédiction et de vengeance. La momie de la BNU s'est-t-elle déjà manifestée de la sorte ? A-t-elle déjà été à l'origine de phénomènes incongrus ?}

Ce n'est pas impossible. Le premier de ces phénomènes inexplicables serait ainsi la disparition de la clef de la caisse dans laquelle elle est conservée et sans laquelle elle ne pourra être examinée de plus près. Ainsi son cercueil demeure scellé et soustrait à notre curiosité. Je n'ai toutefois pas connaissance d'autres malédictions. Mais il est exact que lorsque nous la transportons, nous nous efforçons de la traiter avec tout le respect dû à la dépouille d'un être humain, respect qui va au-delà de celui dû à un document ou à un objet, même archéologique.

\section{Propos recueillis par Julien Collonges}

